
and Systems (ICESS)

\title{
Improving Energy Efficiency and Thermal Comfort of Smart Buildings with HVAC Systems in the Presence of Sensor Faults
}

\author{
Volkan Gunes, Steffen Peter, and Tony Givargis \\ Center for Embedded and Cyber-physical Systems \\ University of California, Irvine, USA \\ Emails: \{vgunes, st.peter, givargis\}@uci.edu
}

\begin{abstract}
The smart building, as an application of the cyberphysical systems (CPSs), plays an important role in everyday lives of people. Thermal comfort and energy efficiency are primary goals for HVAC systems in smart buildings. Since the controllers of the HVACs heavily rely on data of sensors that are deployed in the buildings, temporary or permanent sensor faults may lead to increased energy consumption or decreased thermal comfort far below expectations. In this paper, we examine sensor data faults observed in the real-world sensor deployments, and their effects on thermal comfort and energy efficiency in multi-room buildings. The read-back and nearest neighbor monitoring approaches are proposed considering temporal and spatial correlations between data of sensors to mitigate the faults of interest. We adopt a model-based design methodology for the multi-room building as a CPS application and develop reusable system models in the MATLAB/Simulink environment. We conclude that the aforementioned faults may significantly reduce energy efficiency and thermal comfort unless mitigated. The proposed approaches improved thermal comfort by up to $75 \%$ for the room where the faulty sensor was deployed and reduced total energy consumption by up to $38 \%$.
\end{abstract}

\section{INTRODUCTION}

Embedded and cyber-physical system technologies touch every aspect of our lives. Since most people spend up to $90 \%$ of their time indoors [1], building automation, also known as the smart building, plays an important role in everyday lives of people as a CPS application. Various sensors are deployed in smart buildings to extract data from physical phenomena such as air temperature and quality. Supported by the extracted data, control of a building's heating, ventilation, and air conditioning (HVAC) systems can be achieved through a centralized decision making mechanism. If, however, we assume that the building has multiple rooms and the occupants of each room have different expectations when it comes to the conception of comfort, then centralized control approach for HVAC systems may fail to meet expectations of all occupants.

As a matter of fact, a survey conducted by International Facility Management Association (IFMA) in 2009 shows common HVAC complaints [2]. Received throughout the year, 94\% of common HVAC complaints is regarding ambient temperature being too cold and $91 \%$ of those complaints is regarding ambient temperature being too hot. Among HVAC complaints received in the summer, under air conditioned (i.e., too hot) complaint accounts for $66 \%$ of the complaints and over air conditioned (i.e., too cold) complaint accounts for $58 \%$ of the complaints. The results show that today's HVAC systems cannot adapt to seasonal and daily temperature changes as fast as expected. Besides HVAC related ineffectiveness, indoor air condition may be further aggravated by sensor faults, since reliable HVAC system behavior depends on the data extracted from the phenomenon of interest (i.e., air temperature). These faults may lead to discomfort for occupants and elevated energy consumption.

Distributed control of a building's HVAC systems provides great flexibility to ensure high quality residential comfort and prevent HVAC ineffectiveness in multi-room smart buildings. Moreover, sensor fault mitigation strategies deployed on a multi-room building help enhance the overall HVAC system effectiveness. Therefore, distributed control of a building's HVAC systems and sensor fault mitigation techniques must be leveraged in order to bridge the gap between occupants' expectations and their perceptions of a smart building.

In this study, we examine temperature sensor faults and their impacts on thermal comfort of occupants and HVAC energy consumption. The contributions of this paper are:

- Modeling temperature sensor faults that are observed in the real-world sensor deployments.

- $\quad$ Modeling a multi-room building with HVAC systems by using CPS approach and developing reusable system models in the MATLAB/Simulink environment.

- Providing fault mitigation techniques based on temporal and spatial correlations between sensors' data without the need to replace faulty sensors.

As a result of the proposed approaches, we could improve thermal comfort by up to $75 \%$ for the room where the faulty sensor was deployed and reduce total energy consumption by up to $38 \%$.

\section{RELATED WORK AND MotiVATION}

Various works have studied fault detection and mitigation mechanisms under fault occurrences - in a variety of system scenarios:

A knowledge-based system for sensor fault modeling, detection, and mitigation is presented in [3]. The results are illustrated on an electro-mechanical actuator application and compared to a previously deployed neural network based system. The authors address most common sensor faults such as bias, drift, scaling, and dropout, as well as system faults. 
A multi-kernel approach is proposed in [4] against sensor failures. The approach is aimed at improving the robustness of e-nose systems without the need to replace faulty sensors and repeat system calibrations. The authors validate the proposed approach with actual data from an e-nose system built with 8 metal oxide (MOX) gas sensor array module exposed to six different gases to show that the performance of the system is not affected when increasing the number of faulty sensors.

Timely event detections and and delivering time-critical operations reliably in CPS under sensor fault occurrences are studied in [5]. The authors illustrate sensor fault scenarios with a motion-based object tracking system where the presence of imperfect sensor conditions is likely to cause untimely control action to be taken based on incorrect object position estimation, which in return leads to the system failure.

The design of a model-based methodology is studied in [6] in order to detect and isolate multiple sensor faults in multizone HVAC systems where the interconnected subsystems are characterized by heterogeneous nonlinear dynamics. The authors propose a model-based and distributed architecture for sensor fault detection, which relies on robust analytical redundancy relations.

A fault detection and diagnosis strategy is proposed in [7] using combined neural networks and subtractive clustering analysis to improve energy efficiency and thermal comfort in the buildings. The strategy is aimed at detecting faults such as soft sensor faults (e.g., fixed biases and drifting biases) and complete failure of the sensors in the supply air temperature control loop of HVAC system and removing those faults.

Given the need for thorough understanding of sensor faults' semantics and their effects on the comfort of occupants, we examine temperature sensor faults observed in the real-world deployments and consider a multi-room smart building as a case study. The following section introduces our design methodology and explains temperature sensor fault semantics and terminology used throughout the paper.

\section{Methodology And Fault Semantics}

Smart buildings are CPSs, which are comprised of components such as HVAC systems, sensors, actuators, controllers etc. During the system's lifetime, faults are inevitable for one or many of these components and may have an adverse affect on the system outcome. Therefore, a methodology is required to address faults systematically. In this section, we provide our methodology, a taxonomy of faults, and terminology used throughout the paper.

\section{A. Model-based CPS Design Methodology}

We adopt a model-based approach to design reliable and fault-tolerant CPSs. Model-based design (MBD) methodology allows the designer to design, analyze, verify, and validate CPSs through several consequent design phases. Our modelbased CPS design methodology is shown in Figure 1. A common CPS model consists of the physical system model, the cyber-physical (CP) interface model, and the cyber system model, as represented with green rectangles in Figure 1. The physical system model can be expressed as the description of physical behavior with differential equations. Those equations should reflect both inner dynamics (i.e., plant behavior and conditions) or outer dynamics (i.e., conditions of environment

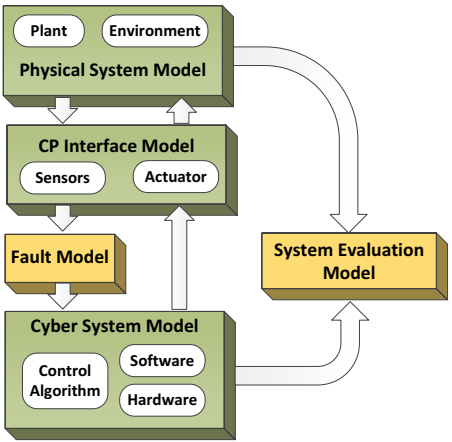

Fig. 1: Model-based CPS design methodology.

surrounding the plant) of the physical system. The cyberphysical (CP) interface model represents the network of sensors and actuators. The cyber system model can be expressed as the control algorithm, and the behavior of software and hardware components of the cyber system. Since our use-case CPS application is a multi-room smart building incorporating temperature sensors, controllers, and HVAC systems, from CPS standpoint, the building, phenomenon of interest, and HVAC systems represent the physical system, the controllers represent the cyber system and the temperature sensors represent the interface between the cyber and physical systems.

In order to evaluate CPS outcome under certain types of sensor faults, we clearly define fault semantics and system evaluation metrics, develop models for sensor faults and system evaluation, and inject those models into the traditional CPS model. By doing so, we aim to examine the behavior of a CPS under imperfect sensor conditions.

\section{B. Fault Terminology and Semantics}

A sensor fault refers to a sensor's reading that is inconsistent with the expected behavior of the physical phenomenon. In order to systematically define the faults for temperature sensors, we examine the semantics of a number of sensor data faults observed in the real-world sensor deployments [8], [9]. The type of faults we analyze are as follows:

- $\quad$ Single-sample-spike (positive and negative spikes)

- $\quad$ Spike-and-stay (positive and negative spikes)

- Stuck-at

Single-sample-spike (SSS) fault refers to a sharp change that randomly occurs on only a single point of the sampled values. The spike may take a positive or negative direction. Spikeand-stay $(\mathrm{SaS})$ fault refers to a sharp change which occurs randomly and subsequently preserves its value for a large number of consecutive points of the sampled values. The spike may take a positive or negative direction. Stuck-at (SA) fault refers to a range of sensor readings that undergo zero rate of change. We assume that the sensors having these faults return to the correct behavior afterwards.

We developed well-defined models of these faults and injected those fault models to the CPS model in accordance with our model-based design (MBD) methodology. We implemented fault generators in Simulink for each type of sensor fault mentioned above. Later in Section VII, we provide the illustrative plots from Simulink models for the abovementioned faults to make the fault semantics more clear. 


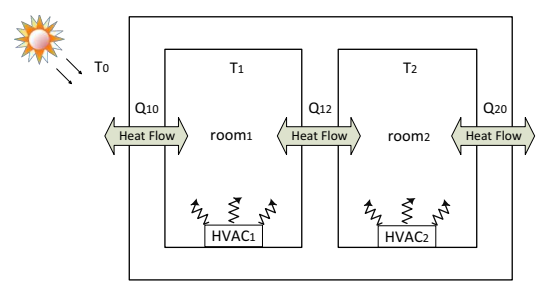

Fig. 2: Heat flows in a building having two rooms.

\section{Multi-Room HVAC Systems}

We investigate multi-room HVAC systems that integrate temperature sensors and controllers as a CPS application. The phenomenon being observed is the room temperature. Our goal is to control room temperatures in a distributed fashion and meet expectations of all occupants with the emphasis on thermal comfort and energy consumption. Each room has a dedicated sensor, controller, and HVAC system. The controllers utilize data of the sensors to turn on/off HVAC systems.

To model temperature variation in the building, a thermal model of the building and HVAC systems are required. Figure 2 shows a sample 2-room building as an illustration of heat flows in the building. $T_{1}$ and $T_{2}$ refer to room temperatures for room $_{1}$ and room $_{2}$, respectively. $T_{0}$ refers to outside temperature. $\mathrm{Q}_{10}, \mathrm{Q}_{20}$ and $\mathrm{Q}_{12}$ represents the heat flows from room 1 to outside, from room 2 to outside, and from room 1 to room $_{2}$, respectively. We describe the thermal behavior of a multi-room building through the laws of thermodynamics. The thermal behavior of entire system is captured by thermodynamic equations [10], which consist of differential and algebraic equations. Same equations are also used to model the effect of hot and cool air supplies provided by HVAC systems on the heat flow of the building. The following section describes the simulation framework and the constituent models for the CPS example and their functionalities.

\section{Matlab/Simulink Simulation FramewORK}

We implemented our MBD approach for the design of a multi-room smart building with HVAC systems ${ }^{1}$ in the MATLAB/Simulink Environment [11]. HVAC systems and thermal properties of whole building are modeled using the aforementioned thermodynamic equations. Figure 3 shows a Simulink model for one room, namely room $_{1}$, incorporating thermal parameters, a temperature sensor, and a controller for the room. Each room in the building has a similar model. In Figure 3, Q Heater1 $_{\text {and }} \mathrm{Q}_{\text {Cooler1 }}$ represent the heat flows supplied by the heating and cooling systems in room $_{1}$, respectively. Since supplied hot air increases the temperature, $\mathrm{Q}_{\text {Heater1 }}$ is added to aggregate heat flow and since supplied cool air decreases the temperature, $\mathrm{Q}_{\text {Cooler } 1}$ is subtracted from aggregate heat flow. $\mathrm{Q}_{12}$ and $\mathrm{Q}_{10}$ represent the heat transfer from room 1 to room $_{2}$ and outside respectively and are subtracted from aggregate heat flow for room 1 . Similar parameters are defined for other rooms as well, following the same notation.

Thermal resistance and capacitance parameters in the model depend on the physical properties of the materials used in the building. Thermal resistance of a material is the function of thickness, surface area, and thermal conductivity of the material and thermal capacitance of a material is the function of mass and heat capacity of the material. In

\footnotetext{
${ }^{1}$ Simulink models are available for download from http://tiny.cc/sb-hvac
}

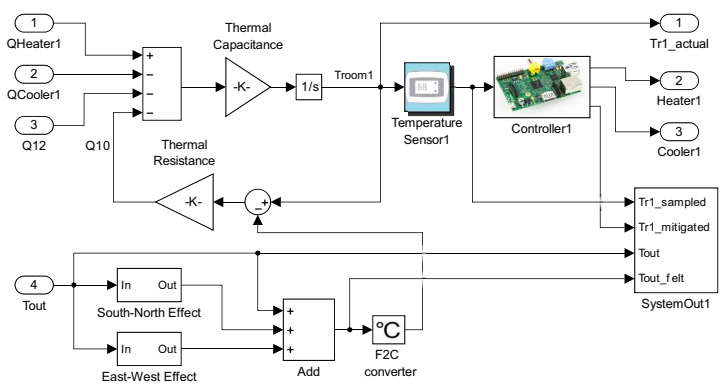

Fig. 3: Simulink model of one room.

Figure 3, Tr1_actual refers to the actual temperature of room $_{1}$. Tr1_sampled refers to data of temperature sensor in room $_{1}$. Tr1_mitigated refers to the temperature data after mitigation technique being applied by the controller for room $_{1}$. The controller processes the sampled temperature data and apply mitigation techniques if any fault is detected. Outputs of the controller carry control commands for the HVAC system's heating and cooling facilities. The HVAC system is controlled by considering the mitigated temperatures.

To have a well-defined thermal model of the building, the effect of direct solar radiation is considered for North, South, East, and West directions and the outcome is referred as Tout_felt. The sun's angle of incidence and the orientation of the room (South/North and East/West) are specified and the result is added to Tout to calculate Tout_felt, which is then used to calculate $\mathrm{Q}_{10}$ as shown in Figure 3 .

\section{A. Fault Model}

The implementation of fault semantics in Simulink with an MBD approach allows us to inject the faults into our system model and analyze their impacts on the overall system behavior. As we mentioned in Section III, we examine some sensor faults observed in the real-world sensor deployments [8], [9]. We provided our terminology and fault semantics for those sensor faults of interest in Section III.

We have a fault model for each type of fault and inject that fault model into the common CPS model complying with our MBD methodology. The system outcome is evaluated under faulty sensor conditions, first without and then with mitigation techniques being applied by the controller.

\section{B. System Evaluation Model}

The system outcome is evaluated based on thermal comfort and energy consumption. In order to evaluate thermal comfort, first we define a comfort interval which is in $10^{\circ} \mathrm{F}$ range. The upper and lower bounds of the range is $5^{\circ} \mathrm{F}$ more and less than the set point (i.e., reference) for the room temperature, respectively. The comfort range indicates, we assume, that the occupants of a room feel comfortable in that temperature interval. The thermal comfort metric is the function of actual room temperature for the corresponding room. The base thermal comfort is assumed to be $100 \%$ in perfect conditions. In faulty sensor conditions, a deduction from the base thermal comfort is calculated according to how long the actual room temperature stays out of the comfort range.

Energy consumption is evaluated by comparing energy consumption of the building under fault occurrences with energy consumption of the building without fault occurrences that is, what we call, the base energy consumption. The 
energy consumption metric is assumed to be $100 \%$ without any fault occurrences. A deduction from the energy consumption metric is calculated considering the difference between the base energy consumption and energy consumption under faulty sensor conditions. The following section explains the proposed mitigation techniques for the aforementioned sensor fault scenarios.

\section{Fault Mitigation Techniques}

Since our main concern in the multi-room building design is to alleviate adverse impact of temperature sensor faults on the occupants' perceptions of a smart building, the temporal and spatial correlations between sensors' readings can be leveraged to design fault-tolerant building automation systems. The following subsections explain the proposed techniques to mitigate the sensor faults of interest in this study.

\section{A. Read-back Technique}

The read-back technique (RBT) considers the temporal correlation between consecutive points of the sensor readings. The rate of change of temperature is observed to identify and mitigate the sensor faults. The rate of change refers to the difference in temperature between two consecutive samples. Since temperature changes slowly, the rate of change of temperature is expected to be low.

In RBT, the controller saves the rate of change for sensor readings and compare it to a threshold value. The threshold refers to the highest observed rate of change under perfect conditions and is calculated through the daily variation pattern of temperature. If the rate of change is above the threshold, then the sampled data is considered to be spike and the previously sampled data is used to control the HVAC system. The designer may predefine the threshold or have the controller learn it through machine-learning techniques.

\section{B. Nearest Neighbor Monitoring Technique}

Since RBT cannot mitigate the faults having zero rate of change, the nearest neighbor monitoring (NNMT) can be applied to mitigate those type of faults. NNMT considers both the temporal and spatial correlations between data of the sensors deployed in two neighbor rooms. In NNMT, the rooms are considered to be the nearest neighbors if they share at least a wall. As in RBT, the rate of change of temperature is observed to identify and mitigate the sensor faults. If the rate of change is below the threshold and not equal to zero then the controller continues using samples from the sensor. However, if it is equal to zero, then that means the sensor is stuck and NNMT is applied to mitigate the fault.

In case of applying NNMT, the controller stops using samples from the faulty sensor till the sensor returns to the expected behavior (i.e., correct behavior of the sensor). Meanwhile the controller samples the temperature data from the sensor in the nearest neighbor room. When the faulty sensor returns to the correct behavior, the controller stops sampling from the nearest neighbor and uses the data extracted by the sensor in the relevant room.

The reason why the temperature data is obtained from the neighbor sensor readings is the spatial correlations between the temperatures in the nearest neighbor rooms. A many core embedded processor like [12] can be used as the control system

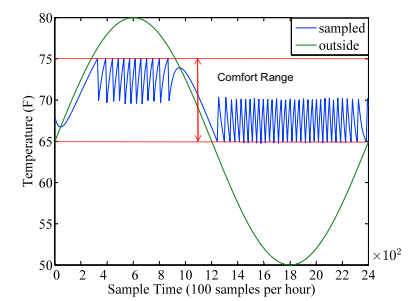

Fig. 4: Room temperature variation in the comfort range.

of the building where each core in the processor represents a controller deployed in a room. The following section discusses our simulation results and system success evaluation before and after mitigation techniques being applied.

\section{EXPERIMENTS AND EVALUATION}

We implemented the proposed fault mitigation techniques in Simulink and applied them to our demonstrative CPS model. As previously mentioned, we define a comfort range considering an upper bound, lower bound, and set point for the room temperature. Figure 4 shows how the temperature of a room changes according to the comfort range specification under perfect conditions. In the figure, the $\mathrm{x}$-axis represents the number of samples taken by the controller throughout a day. The controller takes 100 samples per hour from the sensor. The $\mathrm{y}$-axis represents the temperature in Fahrenheit. The green and blue lines represent the variation of outside temperature and sampled sensor data, respectively.

The set point, upper bound, and lower bound are determined as $70^{\circ} \mathrm{F}, 75^{\circ} \mathrm{F}$, and $65^{\circ} \mathrm{F}$, respectively. They are used to control the cooling and heating facilities of dedicated HVAC system in the room. To be more precise, the cooling system operates between the upper bound and set point and the heating system operates between the set point and lower bound. Since we treat the cooling and heating facilities of an HVAC system as identical heat resources, specifying non-overlapping operation regions averts the deadlock.

Figures 5 and 6 show the temperature pattern of a room throughout a day, considering single-sample-spike (SSS) faults. Figures 5a and $6 \mathrm{a}$ show sampled sensor readings under SSS with positive and negative spikes, respectively. Figures $5 \mathrm{~b}$ and $6 \mathrm{~b}$ show the temperature variation when RBT being applied, referred as mitigated. In the figures, the green, red, and blue lines represent the variation of outside temperature, sampled sensor reading, and mitigated sensor reading, respectively. Since the faults are modeled as occurring randomly, fault patterns are different in Figures (a) and (b). As the results indicate, this technique works well if the fault does not take more than one samples.

Figures 7 and 8 show the variation of room temperature under spike-and-stay ( $\mathrm{SaS})$ fault with positive and negative spikes, respectively. In Figure $7 \mathrm{a}$, the controller initiates the cooling system if the sudden increase in the sampled sensor reading is above the upper bound. The cooling system will operate continuously till the sensor returns to the correct behavior. When the sensor returns to the correct behavior, low room temperature due to over-operation of the cooling system during faulty sensor readings is observed by the sensor. Then, the controller initiates the heater since the room temperature is below the lower bound. Same situation works for the consecutive occurrences of the fault till the end of the day. 


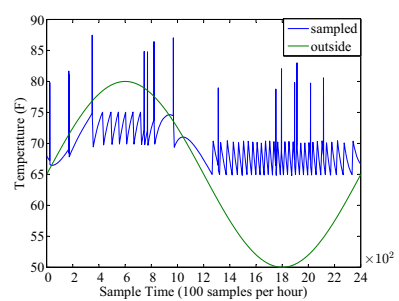

(a)

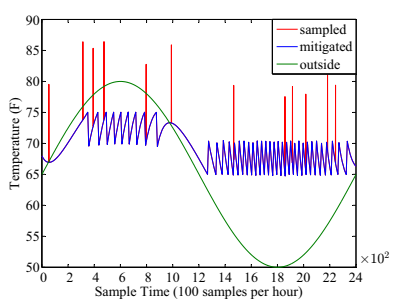

(b)
Fig. 5: Single-sample-spike (SSS) fault with positive spike: a) without mitigation, b) with mitigation through RBT.

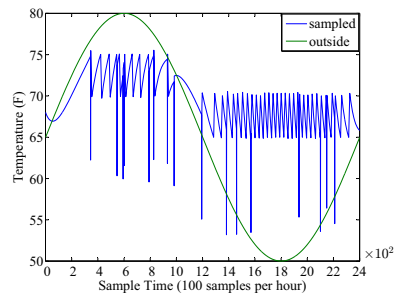

(a)

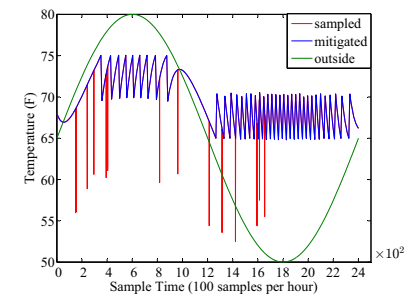

(b)
Fig. 6: Single-sample-spike (SSS) fault with negative spike: a) without mitigation, b) with mitigation through RBT.

In Figure 8a, the controller initiates the heating system if the sudden decrease in the sampled sensor reading is below the lower bound. The heating system will operate continuously till the sensor returns to the correct behavior. When the sensor returns to the correct behavior, high room temperature due to over-operation of the heating system during faulty sensor readings is observed by the sensor. Then, the controller initiates the cooler since the room temperature is above the upper bound. Same situation works for the consecutive occurrences of the fault till the end of the day. Unless $\mathrm{SaS}$ fault is mitigated, it leads to inefficient use of HVACs, increased energy consumption, and occupants' dissatisfaction regarding thermal comfort.

Figure $7 \mathrm{~b}$ shows the variation of room temperature under $\mathrm{SaS}$ fault with positive spike when NNMT is applied. Even tough there is a slight difference in the transitions between sensor readings, the room temperature approximately follows the expected pattern. Obviously, the occurrence of the fault at the time step of around 1800 and its mitigation have different effect than the other ones. That's because this fault occurs while outside temperature is at the minimum (i.e., $50^{\circ} \mathrm{F}$ ) in its daily variation and the nearest neighbor room has more walls shared with outside than the room being observed. The heating facility of the corresponding room is being controlled according to the nearest neighbor readings. So the heater operates more than necessary. When the sensor returns to the correct behavior, the controller realizes that the temperature is slightly beyond the upper bound and the cooler facility is switched on. Considering the temperature variation in the range of 20 to $25^{\circ} \mathrm{F}$ under no fault mitigation, at most $1^{\circ} \mathrm{F}$ temperature difference out of the comfort range in one instance of the fault is favorable.

Figure $8 \mathrm{~b}$ shows the variation of room temperature under $\mathrm{SaS}$ fault with negative spike when NNMT is applied. Likewise positive spike, even tough there is a slight difference in the transitions between sensor readings, the room temperature approximately follows the expected pattern. Obviously, the

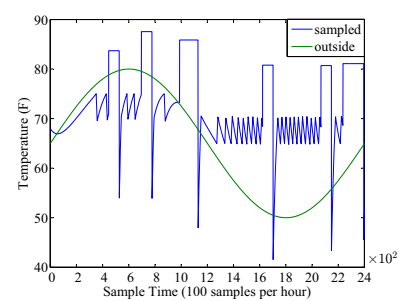

(a)

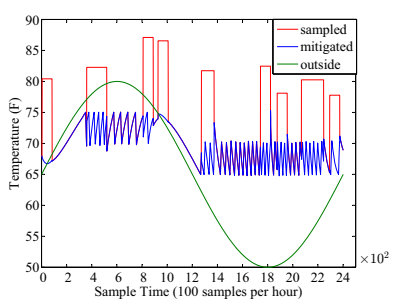

(b)
Fig. 7: Spike-and-stay (SaS) fault with positive spike: a) without mitigation, b) with mitigation through NNMT.

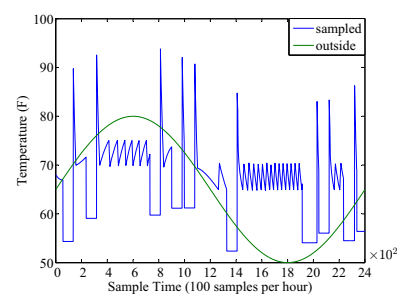

(a)

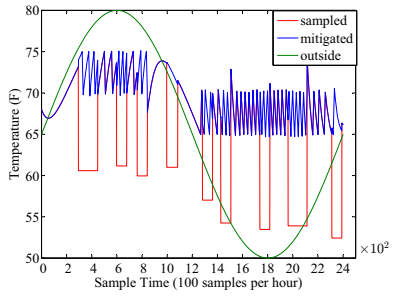

(b)
Fig. 8: Spike-and-stay (SaS) fault with negative spike: a) without mitigation, b) with mitigation through NNMT.

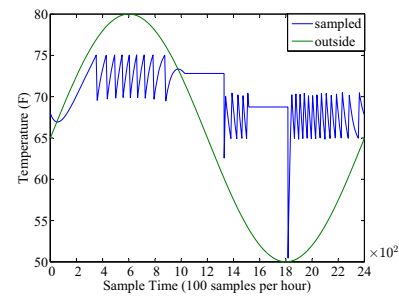

(a)

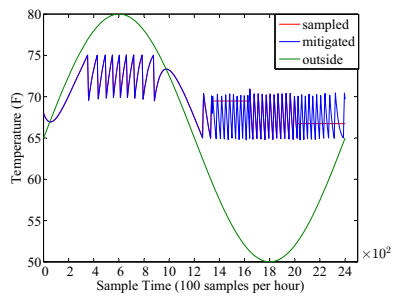

(b)
Fig. 9: Stuck-at (SA) fault: a) without mitigation, b) with mitigation through NNMT.

occurrence of the fault at the time step of around 2000 and its mitigation have different effect than the other ones. The reason is same as we explained in the previous paragraph. Besides, duration of this fault is longer than the other fault occurrences. This causes the heater to operate slightly more than needed. Again, considering the temperature variation in the range of 20 to $25^{\circ} \mathrm{F}$ under no fault mitigation, a few Fahrenheit temperature difference inside the comfort range is favorable.

Figure 9 shows the variation of room temperature under stuck-at (SA) fault occurrences. In Figure 9a, the second instance of the fault occurs when outside temperature goes down below the lower bound. However, since the controller relies on the faulty sensor, it does not initiates the heater. So the room temperature follows the outside temperature and gets very close to the minimum outside temperature (i.e., $50^{\circ} \mathrm{F}$ ) at the end of about 3 hours. When the sensor returns to the correct behavior, low temperature due to under-operation of the heating system is observed by the sensor. Then, the controller initiates the heater since the room temperature is below the lower bound. Figure $9 \mathrm{~b}$ shows the variation of room temperature under SA fault when NNMT is applied. As the figure shows, even tough there is a slight difference in the transitions between sensor data, the room temperature approximately follows the expected pattern. 


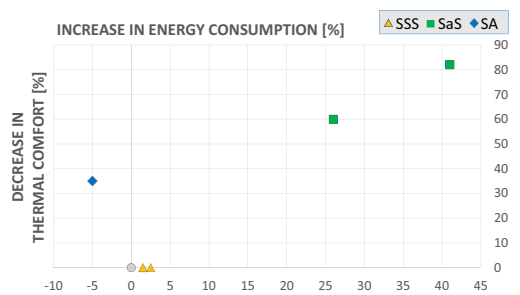

(a)

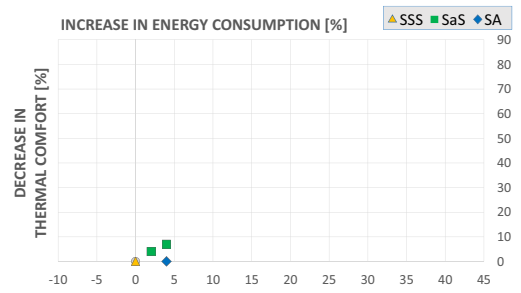

(b)

Fig. 10: Energy consumption and thermal comfort: a) without mitigation, b) with mitigation.

Besides the results regarding room temperature variations, we also provide variation in energy consumption and thermal comfort under faulty sensor conditions without and with mitigation techniques being applied. The summarized results of the experiments are illustrated in Figure 10. The $\mathrm{x}$ axis represents increase in energy consumption as a percentage. The y axis represents decrease in thermal comfort as a percentage. The orange triangles represent single-sample-spike (SSS) faults. The green squares represent spike-and-stay $(\mathrm{SaS})$ faults. The blue diamond represents stuck-at (SA) fault. The gray circle represents the perfect case which indicates zero decrease in thermal comfort and zero increase in energy consumption.

Figure 10a shows that the non-mitigated faults reduce thermal comfort in all cases but SSS faults and increase energy consumption in all cases but SA fault. SSS faults do not decrease thermal comfort because the duration of faults are only single sample but they cause an increase up to $3 \%$ in total energy consumption. Unlike SSS faults, SaS faults cause up to $42 \%$ increase in total energy consumption and up to $83 \%$ decrease in thermal comfort for the room in which the faulty sensor is deployed. The increase in energy consumption is due to over-operation of the HVAC system. The SA fault causes about $5 \%$ decrease in total energy consumption and $35 \%$ decrease in thermal comfort. The decrease in energy consumption is due to under-operation of the HVAC system.

Figure 10b shows the results after the mitigation techniques being applied. By applying the fault mitigation, our goal is to have these data points as close to the gray circle (i.e., perfect case) as possible. The proposed approaches perform very well regarding energy consumption with up to $38 \%$ improvement and thermal comfort of the room with up to $75 \%$ improvement. As the results show, when redundancy is not preferable, the correlation between sensors' data can help mitigate sensor faults and their impacts on the system outcome.

\section{CONCLUSIONS}

Satisfying thermal comfort of occupants and ensuring energy efficiency are important issues in building automation. Since the controllers in the buildings heavily rely on data of temperature sensors to trigger HVAC systems, any occurrence of sensor faults may lead to inefficient use of HVAC systems, elevated energy consumption, and decreased thermal comfort. In this paper, we examined a multi-room building as a CPS application. Given the need for thorough understanding of sensor faults' semantics and their impacts on thermal comfort and HVAC energy consumption, we investigated temperature sensor faults observed in the real-world deployments and provided system evaluation metrics to quantify the impacts of those faults on the system outcome in terms of energy consumption and thermal comfort. We adopted a model-based design methodology for the CPS application and developed reusable system models in MATLAB/Simulink. The modelbased approach enabled us to easily integrate our fault and system evaluation models into the traditional CPS model.

We conclude that the aforementioned faults may significantly increase energy consumption and decrease thermal comfort of the occupants unless mitigated. In order to mitigate the faults of interest, we proposed fault mitigation techniques that are based on the temporal and spatial correlations between sensors' data. The proposed approaches yield up to $38 \%$ improvement in energy consumption and up to $75 \%$ improvement in thermal comfort. In conclusion, the temporal and spatial correlations between sensors' data can be leveraged to design fault-tolerant building automation systems without the need to replace faulty sensors.

\section{ACKNOWLEDGMENT}

This work was supported in part by the National Science Foundation under NSF grant number 1016789 and 1136146.

\section{REFERENCES}

[1] Air and Radiation Basic Information. [Online]. Available: http://www.epa.gov/air/basic.html

[2] "Temperature Wars: Savings vs. Comfort," International Facility Management Association (IFMA), Tech. Rep. [Online]. Available: http://www.ifma.org/docs/surveys/hvacsurvey2009.pdf?sfvrsn=2

[3] J. C. da Silva, A. Saxena, E. Balaban, and K. Goebel, "A knowledgebased system approach for sensor fault modeling, detection and mitigation," Expert Systems with Applications, vol. 39, no. 12, pp. $10977-$ 10989, 2012.

[4] J. Fonollosa, A. Vergara, and R. Huerta, "Algorithmic mitigation of sensor failure: Is sensor replacement really necessary?" Sensors and Actuators B: Chemical, vol. 183, no. 0, pp. 211 - 221, 2013.

[5] V. Gunes, S. Peter, and T. Givargis, "Modeling and Mitigation of Faults in Cyber-physical Systems with Binary Sensors," in the 16th Int'l Conf. on Computational Science and Engineering (CSE). IEEE, Dec 2013.

[6] V. Reppa, P. Papadopoulos, M. Polycarpou, and C. Panayiotou, "A Distributed Architecture for HVAC Sensor Fault Detection and Isolation," IEEE Trans. on Control Systems Tech., vol. PP, no. 99, pp. 1-1, 2014.

[7] Z. Du, B. Fan, X. Jin, and J. Chi, "Fault detection and diagnosis for buildings and hvac systems using combined neural networks and subtractive clustering analysis," Elsevier Building and Environment, vol. 73, no. 0, pp. $1-11,2014$.

[8] A. B. Sharma, L. Golubchik, and R. Govindan, "Sensor Faults: Detection Methods and Prevalence in Real-world Datasets," ACM Transactions on Sensor Networks, vol. 6, no. 3, pp. 23:1-23:39, June 2010

[9] K. Ni et al., "Sensor Network Data Fault Types," ACM Transactions on Sensor Networks, vol. 5, no. 3, pp. 25:1-25:29, May 2009.

[10] H. Klee and R. Allen, Simulation of Dynamic Systems with MATLAB® and Simulink ${ }^{\circ}$, 2nd ed. CRC Press, 2011.

[11] Simulink in R2013a, Mathworks. [Online]. Available: http://www.mathworks.com/products/simulink/

[12] V. Gunes and T. Givargis, "XGRID: A Scalable Many-Core Embedded Processor," in the 12th International Conference on Embedded Software and Systems (ICESS). IEEE, August 2015. 